\title{
CasualLearn: A smart application to learn History of Art
}

\author{
Adolfo Ruiz-Calleja, Miguel L. Bote-Lorenzo ${ }^{[0000-0002-8825-0412]}$, Guillermo \\ Vega-Gorgojo $^{[0000-0002-6916-9213]}$, Sergio Serrano-Iglesias, Pablo García-Zarza, \\ Juan I. Asensio-Pérez ${ }^{[0000-0002-1114-2819]}$, and Eduardo \\ Gómez-Sánchez ${ }^{[0000-0003-0062-916 X]}$ \\ ETSI de Telecomunicación, Universidad de Valladolid \\ Campus Miguel Delibes, Paseo de Belén 15, 47011 Valladolid. \\ \{adolfo@gsic, migbot@tel, guiveg@tel, sergio@gsic, pablogz@gsic, juaase@tel, \\ edugom@tel\}.uva.es
}

\begin{abstract}
Smart Learning promises the connection between formal and informal learning, but how to offer informal learning tasks related to formal learning is still a challenge. This demonstration paper presents CasualLearn, a smart learning application that bridges formal and informal learning to learn History of Art in the Spanish region of Castile and Leon. CasualLearn uses a dataset of 16,221 contextualized informal learning tasks that were semi-automatically created exploiting Open Data from the Web. CasualLearn offers these tasks to students based on their context: their geolocation, the activity they do and the topics covered in their formal education. A demo application is currently available for Android devices.
\end{abstract}

Keywords: Open Data, informal learning, smart learning, ubiquitous learning

\section{Pedagogical background}

Smart Education [1] promises to bridge formal and informal learning experiences. It envisions that learning opportunities from the student's daily life could be related to her formal education, thus obtaining a coherent and personalized learning process. As an example, a student who is learning History of Art may have informal learning experiences when walking around her own town and seeing the historical buildings in it. But how to propose meaningful learning tasks and how to relate them to her formal education is still an open question. One of the problems is that informal learning is typically incidental, self-directed and happens unexpectedly [2]. Hence, it is very difficult to foresee the informal learning opportunities that a learner may come up with and the contexts in which these opportunities may be meaningful [5]. 


\section{Technological background and outlook}

Our approach to overcome the abovementioned problem is to build a collection of learning tasks to be recommended to the learner based on the topics covered in her formal education and her context. In our previous work [3] we semiautomatically created such collection of 16,221 tasks out of data openly available in the Web. More specifically, we imported the description of 2,176 historical buildings of the Spanish region of Castile and Leon from three sources: DBpedia ${ }^{1}$, Wikidata $^{2}$ and the dataset of buildings declared as Assets of Cultural Interest ${ }^{3}$ in the Open Data portal of the regional government. Out of the descriptions of these buildings we applied a set of templates to create our dataset of contextualized learning tasks.

For example, if a church is described in DBpedia as of Romanesque style and is related to the concept modillion, the learning task generator applies a template to create the following task: "The modillion is a typical element of Romanesque style. Find a modillion in this church, take a photo of it and then reflect if you have seen it in other Romanesque monuments. Why?". Then, this learning task is contextualized in the geolocation of the church, is related to certain keywords (e.g., "Romanesque" or "modillion") and a level in the Bloom taxonomy (e.g., "remember"). Another example happens when two religious buildings, one of Gothic style and another one of Romanesque style, are located closer than $1 \mathrm{~km}$. away. In that case, a template creates the task depicted in Figure 2, which invites the student to compare the front door of both buildings. This task is contextualized in the geolocation of the first building, is related to some keywords (e.g., "Romanesque" or "Gothic") and the level "analyze" of the Bloom taxonomy. More details about the data generation process are provided in $[3]$.

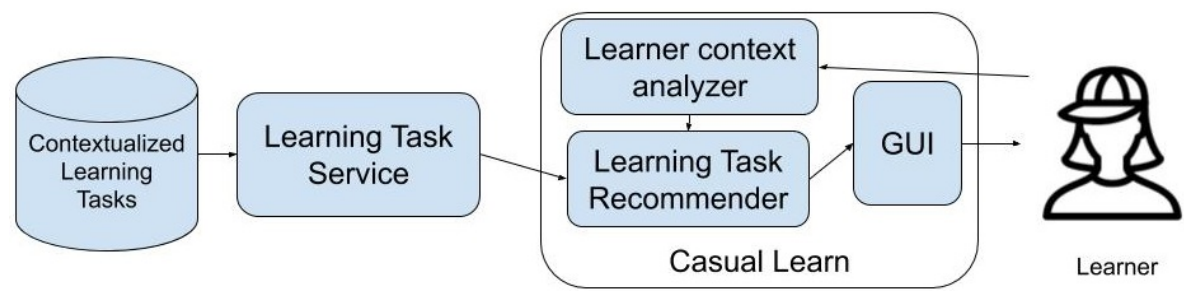

Fig. 1. Software architecture of CasualLearn. Details about Contextualized Learning Tasks and Learning Task Service can be found at [3].

\footnotetext{
${ }^{1}$ http://es.dbpedia.org

2 https://www.wikidata.org/wiki/Wikidata:Main_Page

3 https://datosabiertos.jcyl.es/web/jcyl/set/es/cultura-ocio/ bienes-inmuebles/1284872768044
} 
In this work we present CasualLearn, a content-based and context-aware recommender [5] that exploits this collection to propose meaningful informal learning tasks to students during their daily life. CasualLearn includes three components (Figure 1): a task recommender, a learner context analyzer, and a graphical user interface.

The learner context analyzer maintains the learner's profile and context. In its current version, the learner profile is defined by a set of keywords of interest that the learner introduced (e.g., "Romanesque"). The learner's context is composed of two aspects: the position of the learner and what he is doing. CasualLearn only recommends learning tasks when the learner is walking (i.e., her speed is less than $5 \mathrm{kph}$ ). Knowing the learner's context and her profile, the Learning Task Recommender queries the Learning Task Service to obtain tasks contextualized within a given distance and related to any of the keywords that define the learner's profile. Then, one of the tasks obtained is offered to the learner using the graphical user interface. In this way, our proposal can be considered a content-based and context-aware recommender system [5].

In its current version, CasualLearn supports two main use cases:

- A first use case considers an active learner that searches for tasks to be done. She can visualize the learning tasks available in a map (see Figure 2 left), so she can get closer to the places where they are located. Then, she can actively ask CasualLearn a task for her to carry it out (e.g. Figure 2 right).

- A second use case has been designed for a passive learner that, during her daily life, gets close to the place where a (or some) learning tasks are contextualized. In that case, CasualLearn notifies the student about the task and proposes her to carry it out. If the student rejects the task, CasualLearn will not notify about other tasks for a certain (configurable) amount of time.

CasualLearn is developed for Android devices. The oldest operating system supported is Android KitKat ${ }^{4}$, making it available for $96.2 \%$ of Android devices. The application was developed in Java, using the API Fused Location Provider ${ }^{5}$ to retrieve the geolocalization of the device. CasualLearn uses the maps offered by Open Street Maps ${ }^{6}$. These maps are open licensed and are displayed in the application using the library osmdroid ${ }^{7}$.

\section{Results and outcomes achieved}

We are in the process of evaluating the whole system depicted in Figure 1. Firstly, we are assessing the dataset of Contextualized Learning Tasks. Our preliminary results are promising: 8 teachers assessed 10 learning tasks each and $85 \%$ of them where considered to be useful for secondary school students to learn about

\footnotetext{
${ }^{4}$ https://www .android.com/versions/kit-kat-4-4/

${ }^{5}$ https://developers.google.com/location-context/fused-location-provider

${ }^{6}$ https://www.openstreetmap.org/

7 https://github.com/osmdroid/osmdroid
} 
History of Art. Note that this same infrastructure can potentially be used for creating contextualized learning tasks of other domains, such as Art, Forestry or Medicine.

In the near future we plan to use CasualLearn in authentic settings. More specifically, we will use CasualLearn in two secondary schools located in two different cities of Castile and Leon. Thus, we will assess the usability of the application, its acceptability and the impact of the learning tasks proposed by CasualLearn to the formal education.

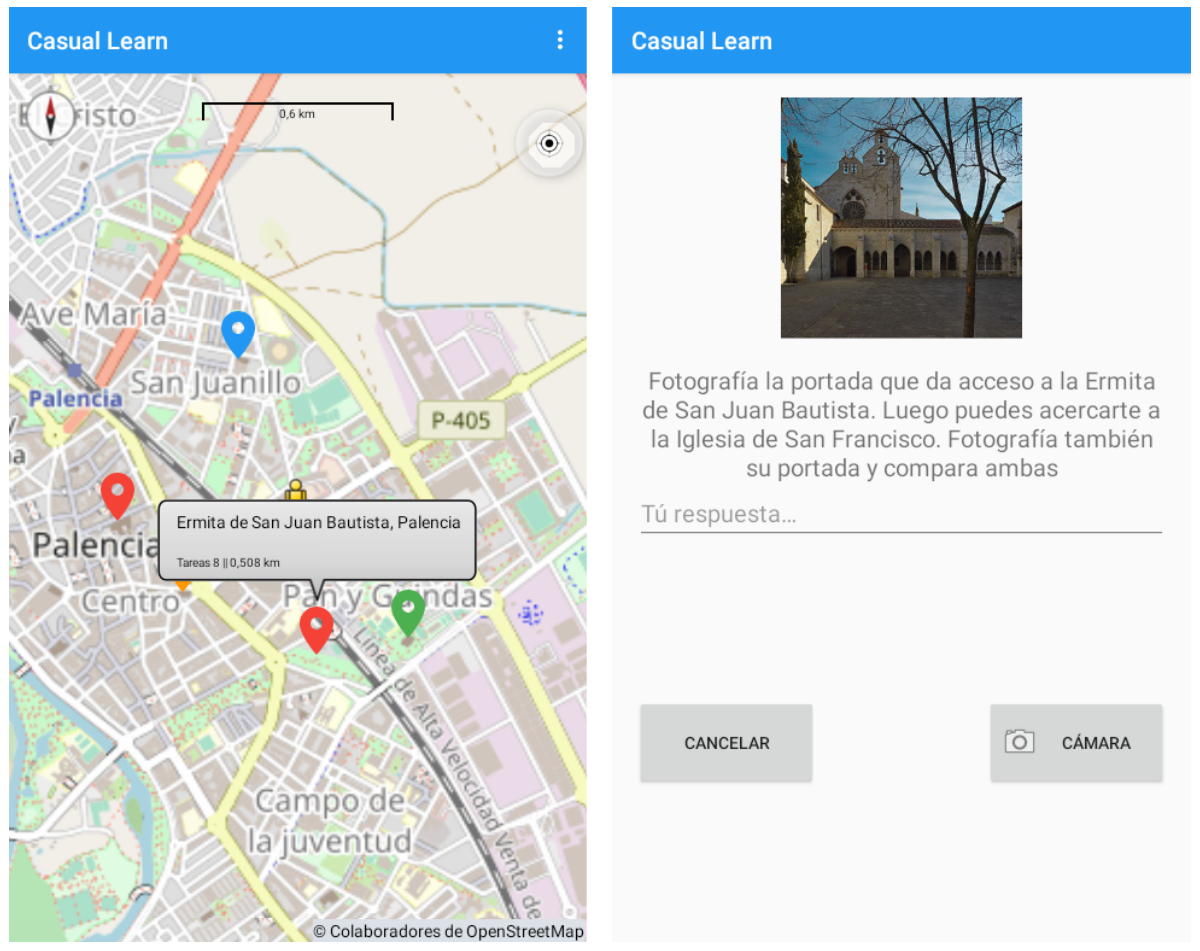

Fig. 2. CasualLearn GUI snapshots: map view interface (left) and task interface (right). The text in the task interface says: "Take a photo of the portal of the Hermitage of Saint John. Then, you can go to the Church of Saint Francis. Take another photograph of its portal and compare both of them."

In our future work we plan to integrate CasualLearn into a Smart Learning Environment [4], also under development. We aim the SLE to inform the task recommender about the learner model, and the characteristics of the learning designs she is participating in within her formal educational context. Hence, the topics of interest will be automatically extracted from the learning design, and filtered by her profile, which is continuously being updated in the formal context. 
We also plan to develop a task authoring tool, so teachers can include new tasks to CasualLearn.

\section{Acknowledgements}

This research has been partially funded by projects VA257P18 (Regional Government of Castile and Leon, ERDF), TIN2017-85179-C3-2-R (AEI, ERDF), and 588438-EPP-1-2017-1-EL-EPPKA2-KA (European Commission).

\section{References}

1. B. Gros. The design of smart educational environments. Smart Learning Environments, 3(15):1-11, 2016.

2. V. J. Marsick and K. E. Watkins. Informal and incidental learning. New directions for adult and continuing education, 2001(89):25-34, 2001.

3. A. Ruiz-Calleja, M. L. Bote-Lorenzo, G. Vega-Gorgojo, S. Serrano-Iglesias, J. I. Asensio-Pérez, Y. Dimitriadis, and E. Gómez-Sánchez. The potential of open data to automatically create learning resources for smart learning environments. Proceedings of the 13th International Conference on Ubiquitous Computing and Ambient Intelligence UCAmI 2019, 2019. 31, 61.

4. S. Serrano-Iglesias, M. L. Bote-Lorenzo, E. Gómez-Sánchez, J. I. Asensio-Pérez, and G. Vega-Gorgojo. Towards the Enactment of Learning Situations Connecting Formal and Non-Formal Learning in SLEs. In International Conference on Smart Learning Environments (ICSLE 2019), pages 187-190, Denton, Texas, USA, 2019.

5. K. Verbert, N. Manouselis, X. Ochoa, M. Wolpers, H. Drachsler, I. Bosnic, and E. Duval. Context-aware recommender systems for learning: A survey and future challenges. IEEE Transactions on Learning Technologies, 5:318-335, 102012. 